\title{
Bariatric surgery during COVID-19 in the UK: a British Obesity and Metabolic Surgery Society (BOMSS) survey
}

\author{
Ahmed Ghanem ${ }^{1,2}$ (1) $\cdot$ Sameh Emile ${ }^{2} \cdot$ Jonathan Cousins ${ }^{1} \cdot$ David Kerrigan $^{3} \cdot$ Ahmed Rashid Ahmed $^{1}$
}

Received: 10 September 2020 / Accepted: 9 January 2021 / Published online: 19 February 2021

(c) Springer Science+Business Media, LLC, part of Springer Nature 2021

\begin{abstract}
Background After the declaration of COVID-19 as a pandemic last March 2020, several adjustments in surgical services were implemented. Plans are now being formulated for restarting bariatric surgery. The aim of this survey is to capture the practice during the pandemic and the readiness to restart to provide a framework to deal with the backlog of bariatric cases. Method A survey was delivered to consultant surgeon members of the British Obesity and Metabolic Surgery Society and non-bariatric surgery consultant members of the Association of Upper GI Surgeons.

Results The survey elicited a response rate of $40 \%(n=66)$ among bariatric surgeons and $15.5 \%(n=34)$ between non-bariatric surgeons. The average question response rate was $93 \%$ (88-100\%). Most of the elective bariatric surgeries and clinics were cancelled early after declaration of the pandemic. Remote technologies for patient education evolved and were used heavily during the pandemic. The average cancelled elective bariatric surgery operations per week was 9 . Nearly a quarter of responders reported performing emergency bariatric surgery during the pandemic. Most of the bariatric surgeons reported being ready to restart the service within 1-2 months. Responders recommended using private sector beds to increase NHS capacity and using the link between obesity and poor COVID-19 outcomes to push for prioritisation of bariatric patients. Conclusion This survey is an attempt to understand the impact of COVID-19 on UK bariatric service and the preparedness to restart. It expressed the bariatric surgery consultants' view of prioritisation of bariatric patients on clinical basis rather than the first-come-first-served basis.
\end{abstract}

Keywords COVID $\cdot$ Obesity $\cdot$ Bariatric $\cdot$ Laparoscopy $\cdot$ Survey $\cdot$ BOMSS

Currently there is no controversy that obesity is associated with multiple comorbidities impairing quality of life and decreasing life expectancy $[1,2]$. Results of medical treatment for obesity have been disappointing so far, therefore

Ahmed Ghanem

a.ghanem1st@gmail.com

Sameh Emile

sameh200@hotmail.com

Jonathan Cousins

johncousins@me.com

David Kerrigan

David.Kerrigan@phoenix-health.co.uk

Ahmed Rashid Ahmed

ahmed.ahmed4@nhs.net

1 Imperial College Healthcare NHS Trust, London, UK

2 Mansoura University Hospital, Mansoura, Egypt

3 Phoenix Health, Merseyside and Lancashire, UK bariatric surgery has been considered the most effective treatment for morbid obesity [3].

On 31 December 2019, the World Health Organization (WHO) reported that a novel coronavirus "SARS-CoV-2" was identified in Wuhan Municipal Health Commission, China where a cluster of cases of pneumonia were reported. COVID-19 was declared as a pandemic by the WHO on the 11th March 2020 due to its alarming levels of spread over a wide geographic area affecting an exceptionally high proportion of the population [4].

After the pandemic status declaration, several adjustments in surgical services were made to cope with the predicted increased demand of intensive therapy units (ITU) and medical wards. NHS England informed hospitals to postpone elective surgical procedures for three months starting on 15th April 2020. The Royal College of Surgeons (RCS) recommended that where appropriate, hospitals and surgical teams should aim to deliver virtual outpatient appointments 
by video link, email or telephone for the duration of the COVID-19 outbreak to support infection control [5].

Plans are now being made for the restart of elective surgery. Given the special medical and cultural aspects regarding obesity and bariatric surgery, there was a concern that bariatric surgery patients may well by disadvantaged by this process.

This survey of UK bariatric consultant surgeons is an attempt to report on surgeons' readiness and preparedness to restart bariatric practice and aims to provide a framework to deal with the backlog of bariatric cases that has built up since the pandemic started.

\section{Materials and methods}

\section{Rationale for the survey}

This survey was implemented to capture the current situation of bariatric surgery practice amid the COVID-19 pandemic and to provide a framework to deal with the backlog of bariatric cases that has built up since the pandemic has started.

\section{Targeted audience of the survey}

Consultant surgeon members of the British Obesity and Metabolic Surgery Society (BOMSS) and non-bariatric surgery consultant members of the Association of Upper GI Surgeons (AUGIS).

\section{Process of designing the survey}

At the time of the survey there were no guidelines for managing patients with morbid obesity during and after COVID19 , and so the survey questions were designed based on NHS and Royal College of Surgeons recommendations for elective surgeries [6], twitter hashtag \#bariatric and \#obesity and public news.

A series of 28 questions were designed. The questions covered enquired surgeons' experiences and bariatric surgery practice during the COVID-19 pandemic and their plans for managing the backlog of bariatric patients during and after COVID-19 time. The survey was designed by bariatric surgery consultants in conjunction with a bariatric surgery fellow and a bariatric anaesthetist. After designing the survey, it was reviewed and amended by BOMSS council before going live. An electronic invitation was sent by email to the consultant surgeon members of BOMSS and AUGIS to complete the survey. The invitation explained the proposed questionnaire and included a link to an online survey. The survey was set up using SurveyMonkey ${ }^{\circledR}$ and went live on 30 April 2020 and closed on 8th May 2020.
Data analysis was done using SPSS version 22, data were described as mean and standard deviation (SD) or median and range for continuous variables and numbers and proportions for categorical variables. Chi-square test was used for statistical analysis of data. $P$ values less than 0.05 were significant.

\section{Results}

The survey was sent to 164 bariatric surgery consultants (BOMSS) and to 220 non-bariatric surgery consultants (AUGIS). A total of 120 consultants opened the survey link and 100 answered the survey. The average question response rate was 93\% (88-100\%) (Fig. 1).

Non-bariatric surgeons were only asked about their view regarding the restart of elective bariatric surgery, commissioning criteria for bariatric surgery, and prioritisation of bariatric surgery.

\section{Elective bariatric surgery}

While cancelling of elective bariatric surgery started as early as the third week of January 2020, some of our responders reported operating till the first week of April 2020 (Fig. 2).

97\% of bariatric surgeons reported stopping bariatric outpatient clinics for new and follow-up cases, mainly in the period $10 / 03 / 2020$ to $20 / 03 / 2020$. Some $73 \%$ of surgeons used video/telephone conferencing to continue outpatient activity whilst physical consultations were halted (Fig. 3).

\section{Types of responders}

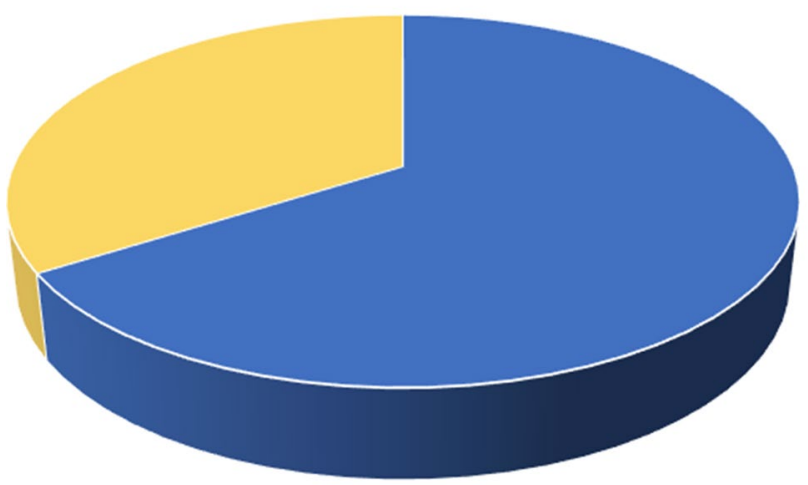

- Bariatric surgeons: group 1 " Non-bariatric surgeons: group 2

Fig. 1 Types of responders 
Fig. 2 Majority of elective bariatric surgeries were cancelled from mid-March 2020
Date of stoppage elective bariatric surgery

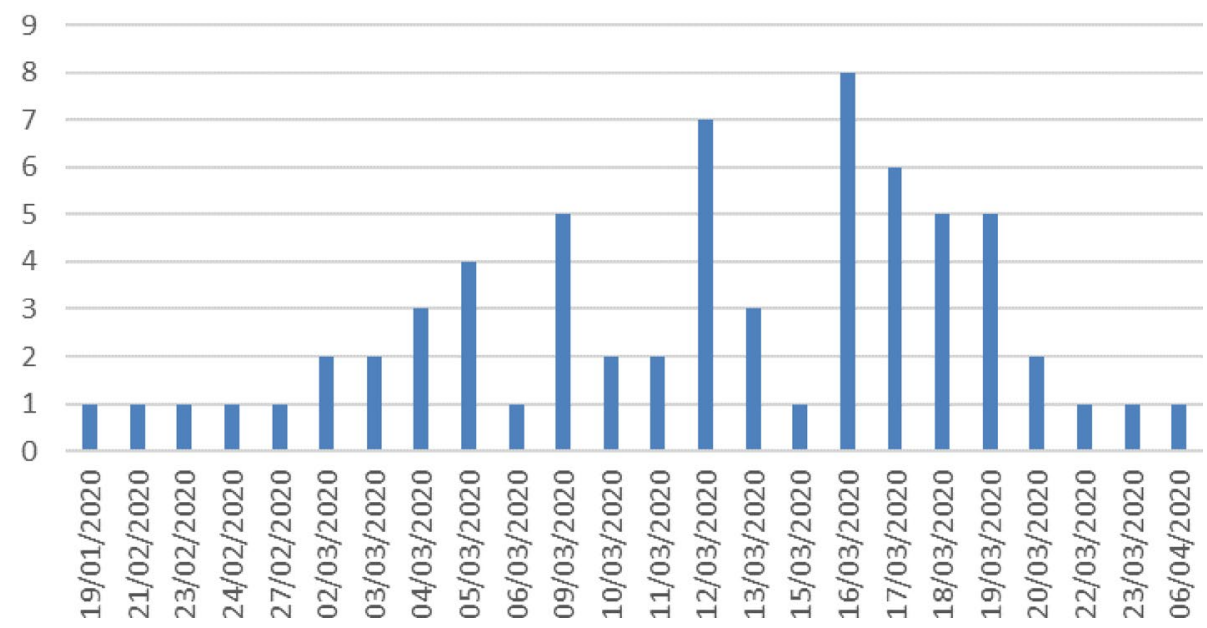

\section{Activity of bariatric out-patient clinic}

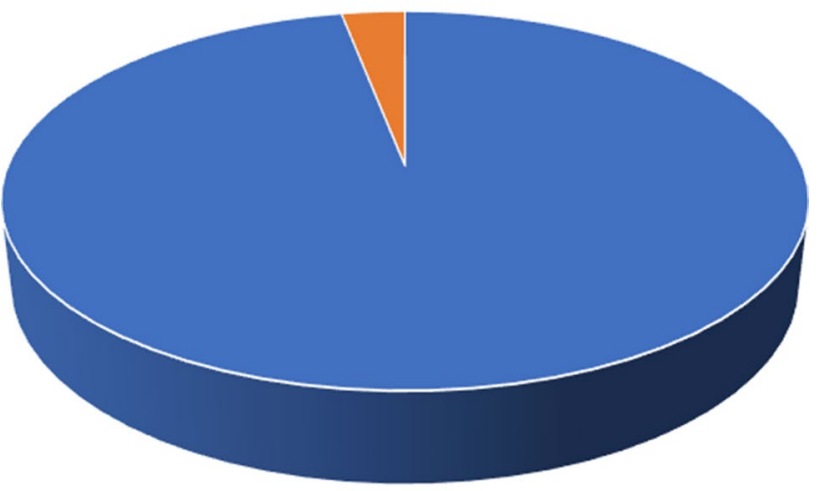

- New and follow-up $=97 \%$ New only $=3 \%$

\section{Remote technologies for patient education}

Remote technologies as video conferencing and web links were used by one-third of the responders to communicate with the patients during the pandemic (Fig. 4).

\section{Tier 3 service and MDT}

Less than one-tenth of responders reported their local bariatric tier 3 service was running during the peak of COVID19 , and only a quarter of bariatric multidisciplinary teams (MDTs) continued to discuss both new referrals and postoperative problems (Fig. 5).

In England, patients need to successfully complete a certain pathway through sequential tiers before being considered for surgery in tier 4 (Table 1) [7].

Fig. 3 Reporting the activity of the outpatient clinic

Fig. 4 Using remote technology for patient education during and after COVID-19 pandemic
Are you planning to continue using remote technology for patient education after COVID-19 pandemic ends?

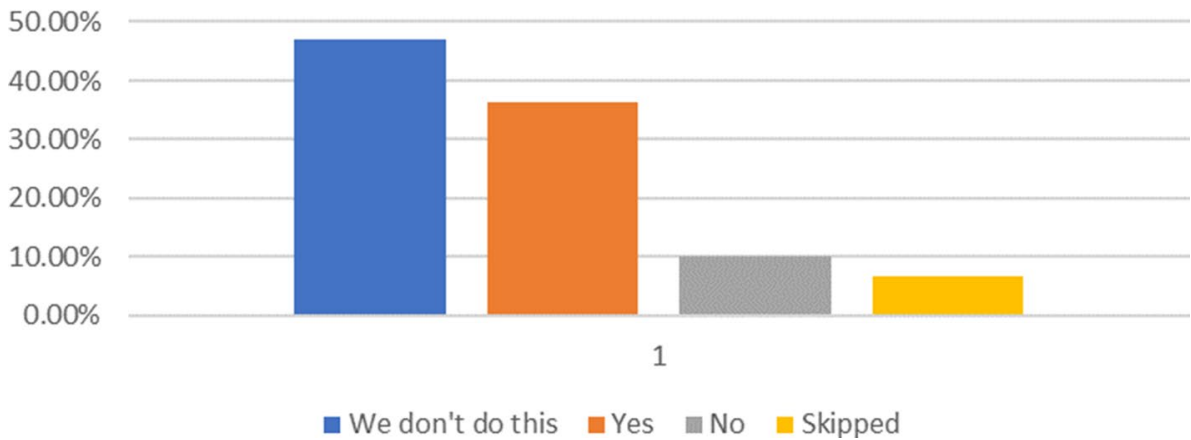


Bariatric surgery waiting lists seemed to be affected as more than half of the responders stopped adding new patients to the waiting list (Fig. 6).

Responders from 22 bariatric units around the UK reported an average of 9 elective bariatric surgery operations per week (range 2-18) to be cancelled. From individual perspective, an average of 4 (range 1-15) cases per week per surgeon were reported to be cancelled due to current COVID-19 situation (Fig. 7).

While the majority reported seeing less bariatric emergency referrals compared to pre-COVID-19, almost a quarter of bariatric surgeons had performed emergency bariatric surgery since elective surgery stopped, $80 \%$ of which were undertaken laparoscopically. (Fig. 8).
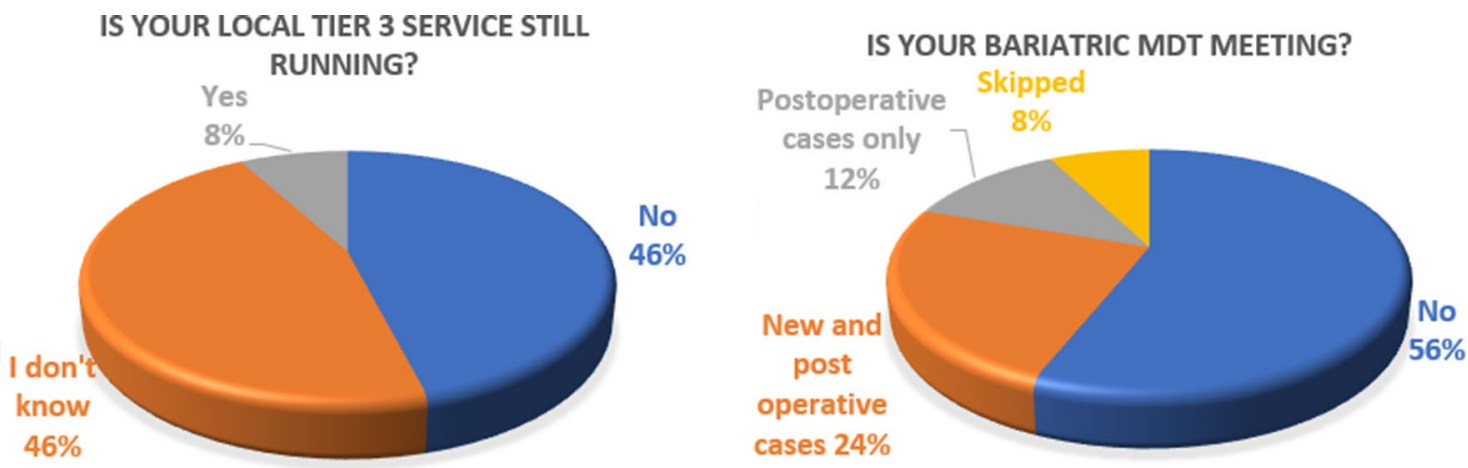

Fig. 5 Almost half of tier 3 service (right-sided pie chart) were stopped and more than half of bariatric MDT meetings were not held (left-sided pie chart) during COVID-19 peak

Table 1 Explaining the different tiers of care for patients with morbid obesity

Fig. 6 Demonstrating bariatric surgery waiting list during the pandemic
Tier 1 Public health programmes aimed at preventing and improving physical activity and healthy eating

Tier 2 Lifestyle weight management advice. Usually given in primary care

Tier 3 One to one management by a medically qualified specialist in obesity. It includes physical activity, psychological and specialist dietetic input in addition to full range of medical specialists as required for comorbidity management

It can be either community or hospital based

Tier 4 Specialist obesity medical and surgical MDTs provide one-to-one management with full access, as needed, to a full range of medical specialists

Patients will be referred to tier 4 by tier 3 . A qualitative level of expertise in patient care makes the difference between a medical specialty at levels 3 and 4

All surgical procedures will take place in tier 4

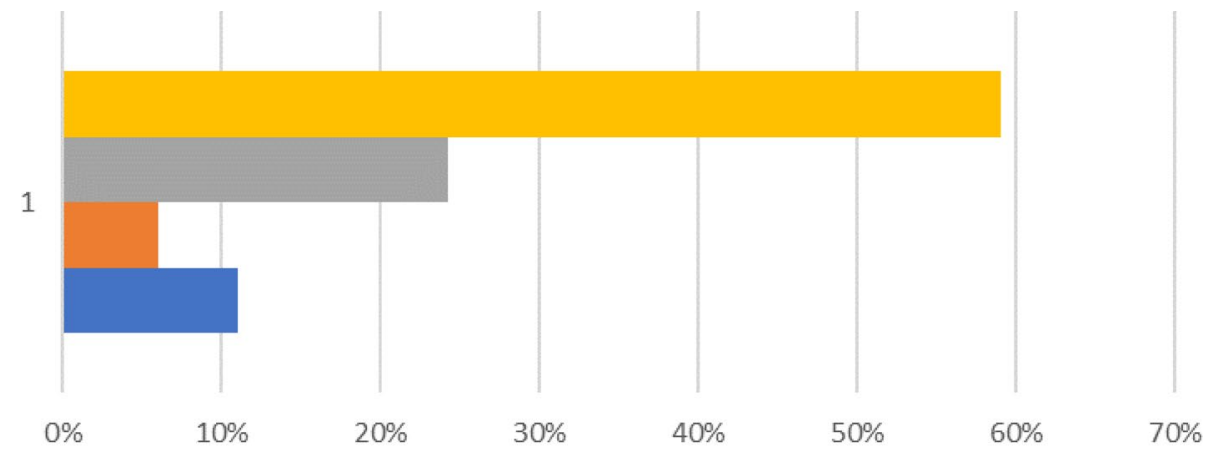

- Stopped adding new patients

늘 Still adding new patients

- Stopped adding and discharged patients back to GP

Didn't prefer to respond 


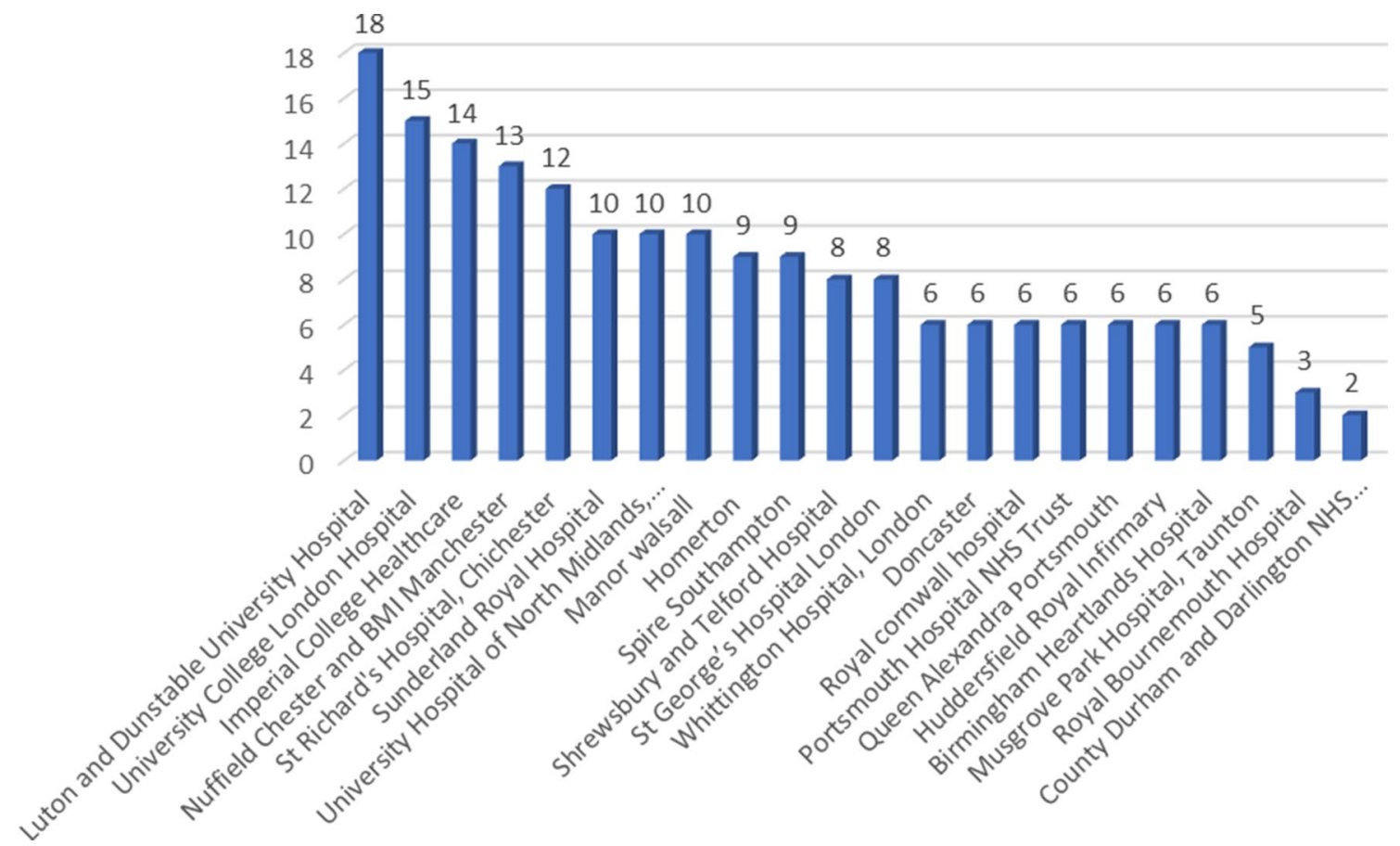

Fig. 7 Number of cancelled bariatric surgeries per week due to COVID-19

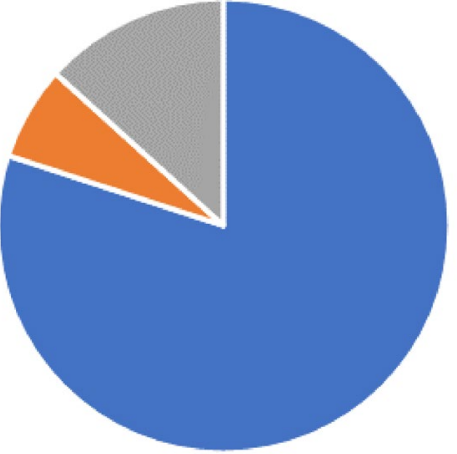

- Laparoscopic = Open ( Both

Fig. 8 How was emergency bariatric surgery performed?

Only $8 \%$ of surgeons were aware of situations where patients had come to harm due to complications of bariatric surgery because the patient did not seek help because of COVID-19 or help has been sought and either 111, primary care or secondary care who had not made a referral on for specialist bariatric advice.

Some of the reported complications included slipped band, intragastric balloon spontaneous rupture, and a patient needing emergency band de-fill who could not access care locally because of COVID-19 and subsequently had to travel to another unit for urgent band removal two weeks later.
When bariatric surgeons were asked how BOMSS should help ensure bariatric surgery is not relegated to low priority status post COVID-19, most bariatric surgeons recommended using the link between obesity and poor COVID-19 outcomes to push for prompt priority treatment of bariatric patients and also advocated using private sector beds to increase NHS capacity. Other recommendations included: (i) specifying a COVID-19-free-area in each trust to operate, (ii) compare long-term survival outcomes for bariatric surgery patients to those of cancer surgery patients, (iii) stratify bariatric patients and prioritise those in utmost need for surgery (Fig. 9).

Discrepancy was obvious when asking about commissioning criteria to access bariatric surgery. Using Chi-square test, there was statistically significant difference between bariatric surgeons and non-bariatric surgeons regarding commissioning criteria for bariatric surgery (Fig. 10, Table 2).

The same statistically significant attitude was reported regarding the priority of bariatric surgery between bariatric and non-bariatric surgeons (Fig. 11, Table 3).

When bariatric surgery restarts, the majority of bariatric surgeons $(61.5 \%)$ would give highest priority to those patients suffering postoperative complications. $30 \%$ would give priority to type 2 diabetics and $21 \%$ to patients needing bariatric surgery as a bridge to other treatments. High BMI ranked lowest with only $9 \%$ of surgeons giving priority to this group (Fig. 12). 
Fig. 9 How to ensure bariatric surgery is not relegated due to low priority status
Push for prompt priority treatment of bariatric patients

- Promote use of private sector beds

\section{Canvas the NHS to increase commissioning of NHS Bariatric surgery. \\ New criteria for prioritizing Bariatric patients \\ Do nothing}

- Other

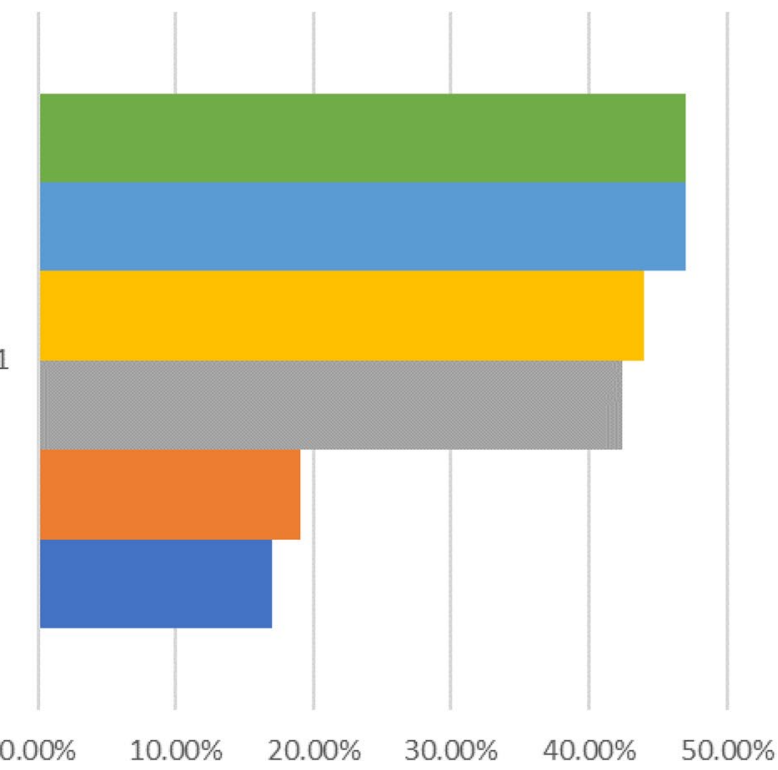

Fig. 10 Bariatric surgeons reported bariatric surgery as a high/medium priority, while non-bariatric surgeons reported the opposite

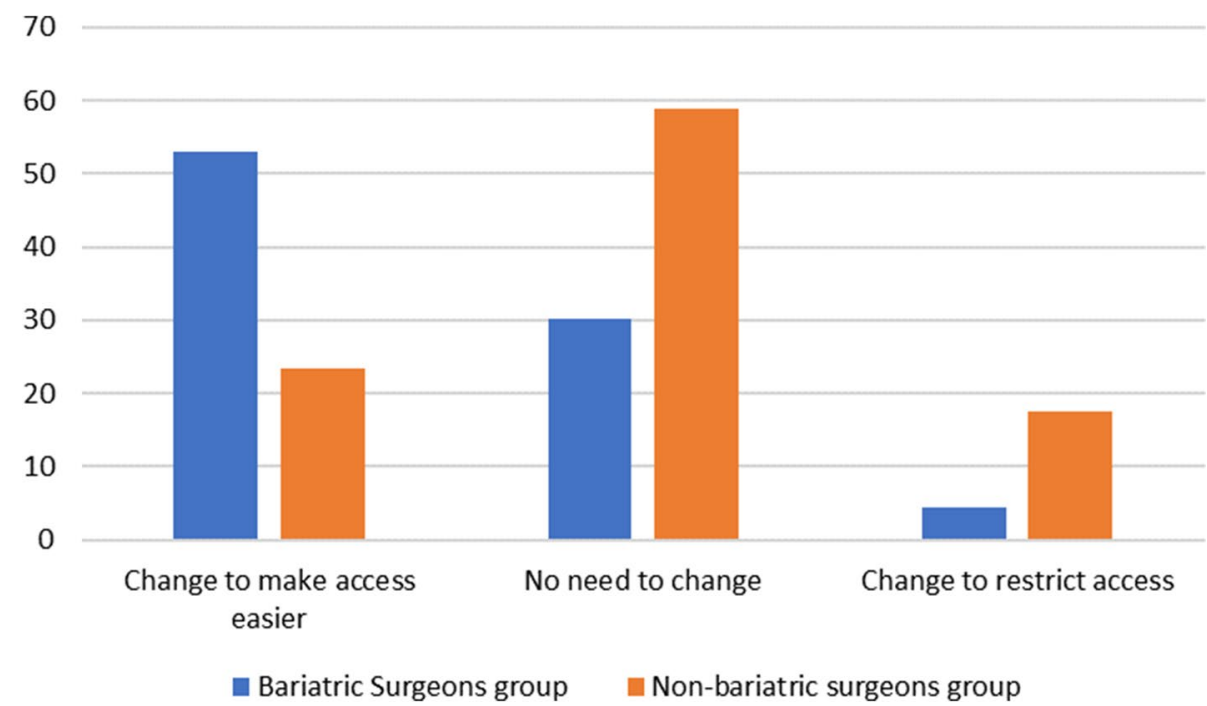

Training programme for bariatric fellows

Table 2 Showing statistically significant difference between two groups

\begin{tabular}{llll}
\hline Variable & $\begin{array}{l}\text { Bariatric } \\
\text { surgeons }\end{array}$ & $\begin{array}{l}\text { Non-bariatric } \\
\text { surgeons }\end{array}$ & $P$ value \\
\hline Change to make access easier & 35 & 8 & 0.0018 \\
No need to change & 20 & 20 & \\
Change to restrict access & 3 & 6 & \\
\hline
\end{tabular}

\section{Readiness to restart bariatric surgery}

More than half of bariatric surgeons reported being ready to restart the service within 1-2 months (Fig. 13).
There were no plans to compensate for the loss of training for bariatric registrar/fellow as reported by the majority of responders.

\section{Discussion}

The COVID-19 pandemic has had unprecedented effects on global healthcare systems, employment, and the economy. To date, there have been well over 400,000 deaths due to this virus worldwide [8].

Recommendations from the NHS early on in the pandemic advised against non-life-saving surgery including bariatric surgery [9]. The reasons for this were: (i) 
Fig. 11 Bariatric surgeons reported bariatric surgery as a high/medium priority, while non-bariatric surgeons reported the opposite

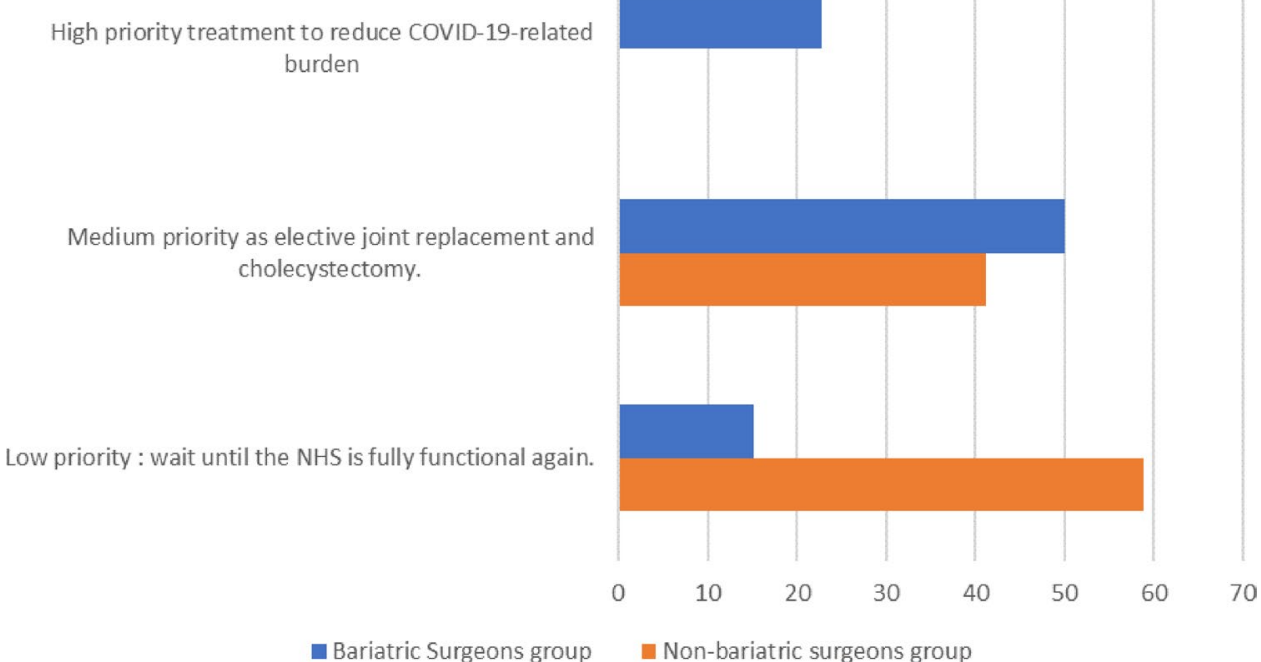

- Bariatric Surgeons group

Non-bariatric surgeons group

Table 3 Illustrating the statistically significant difference in bariatric surgery priority between the two groups

\begin{tabular}{llll}
\hline Priority & $\begin{array}{l}\text { Bariatric sur- } \\
\text { geons }\end{array}$ & $\begin{array}{l}\text { Non-bariatric } \\
\text { surgeons }\end{array}$ & $P$ value \\
\hline High & 15 & 0 & 0.000025 \\
Medium & 33 & 14 & \\
Low & 10 & 20 & \\
\hline
\end{tabular}

perceived increased risk of nosocomial infections (ii) perceived increased risk of complications including mortality by operating on asymptomatic COVID-19 cases and (iii) the need to keep in-hospital bed capacity prioritised for COVID-19 patients as well as more urgent cases such as oncology surgery patients. On the other hand, the risks of delaying surgical treatment for patients with morbid obesity and/or metabolic diseases are very high and should
Fig. 12 Prioritisation of patients for bariatric surgery

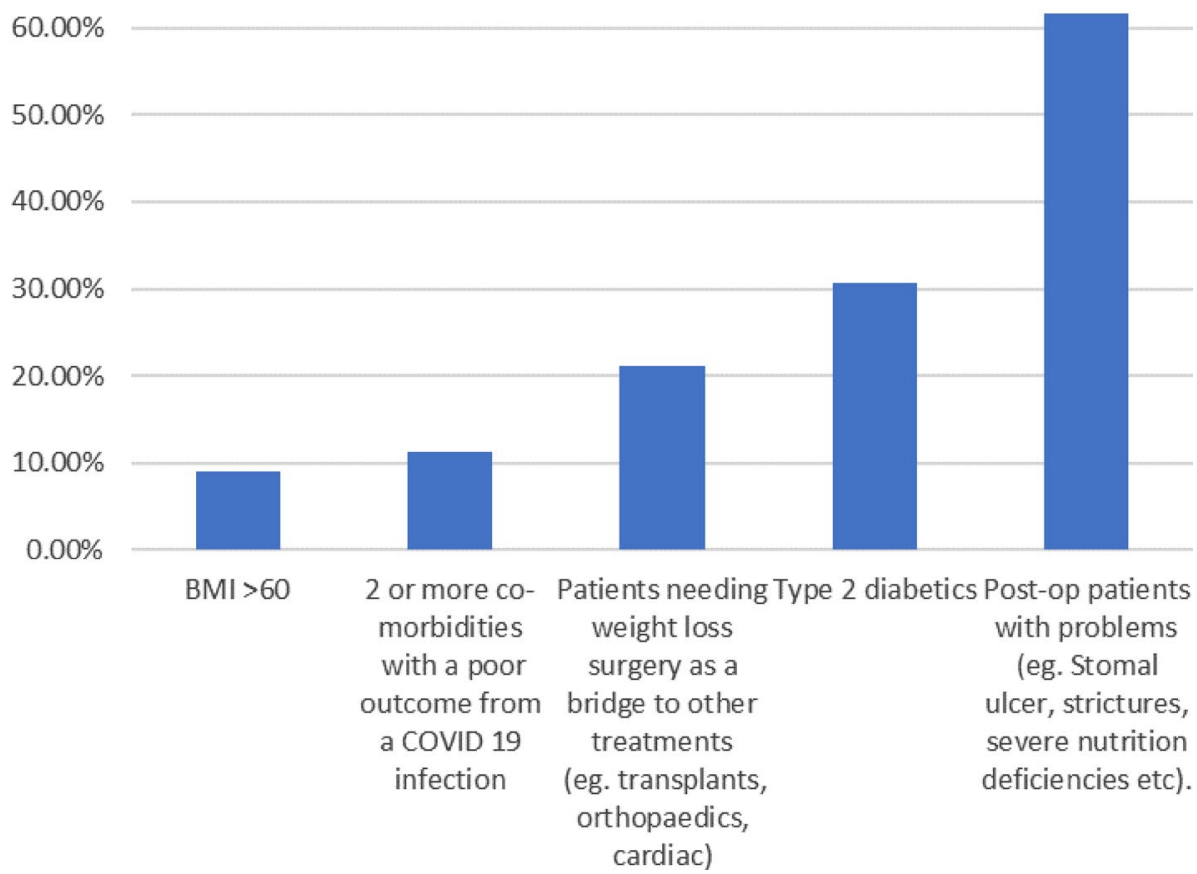


Fig. 13 Time needed to restart bariatric surgery

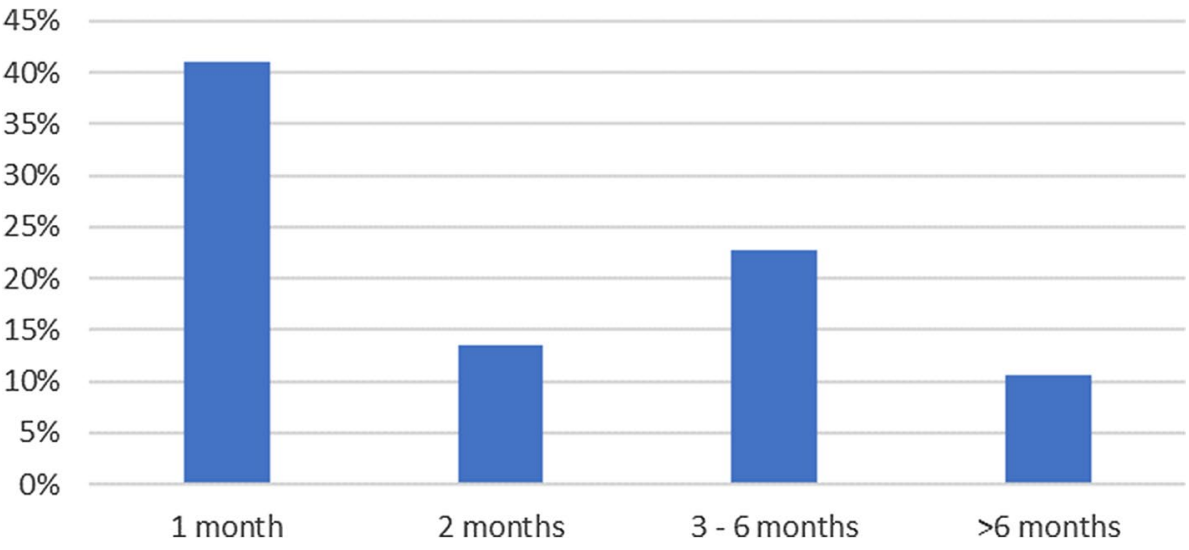

As an alternative to direct face-to-face outpatient clinics, most surgeons were able to use tele-conferencing as a surrogate to in-person outpatient activities. Indeed, telemedicine has been described as a convenient way to connect doctors and their patients in disasters and public health emergencies as it is well suited for scenarios where the infrastructure remains intact and clinicians are available to see patients [18]. Its reported that in March 2020 the proportion of telephone appointments increased by over 600\% [19].

Bariatric MDT meetings have mostly ceased, except in a few units that continued MDT discussions to help patients with postoperative problems. Virtual MDT meetings have been described in the field of oncology amid the current COVID-19 pandemic. The virtual MDT meeting can be held via video conference with one person assigned as a coordinator with other MDT members [20]. Therefore, it maybe plausible and more practical to resume MDT meetings on the virtual platform even after the resolution of the pandemic. MDT meetings were not automatically transitioned to a tele-platform at the time of this survey and the possible cause for this is the absence of common secure platform approved by the NHS to discuss patients, but now after the introduction of Microsoft teams, this can be easily done or even can replace the physical MDT.

Tier 3 lifestyle programme services which normally feed into tier 4 bariatric surgery services have also been affected by the pandemic. Although this is unlikely to have a significant effect on bariatric activity when it restarts as almost all units have enough cases on their existing waiting lists, stopping tier 3 service will affect the progression of obesity related comorbidities and quality of life [21]. Dietary and/or pharmacological strategies for weight loss can be adopted as an alternative for patients with weight-responsive comorbidities who may have to wait long for bariatric and metabolic surgery, depending on the evidence regarding their efficacy $[22,23]$.

Most bariatric units stopped adding new patients to waiting lists for bariatric surgery. An average of four bariatric cases per week were cancelled in 23 bariatric surgery units 
in the UK that responded to the survey. This implies that over the months of March and April, at least 736 patients in the UK would have had their bariatric surgery cancelled. Encouragingly, the majority of surgeons, given the opportunity, could restart bariatric surgery within 1 month of the recommencement of elective surgery. However, the impact of elective surgery cancellation has been studied and statistical analysis shows that even when the normal surgical volume is increased by $20 \%$, a median of 45 weeks will be needed to clear the backlog of operations [24].

As compared to March 2019, emergency attendances have dropped by approximately 44\% during March 2020 which indicates that fewer patients are seeking help for emergencies [25]. The findings of this survey are consistent with bariatric emergency operations being performed less than before COVID-19. This may be explained by the fewer primary operations being performed after the onset of COVID19 pandemic; hence one would expect fewer postoperative complications such as leaks and/or bleeds. But this still does not explain the low rates of patients presenting with slipped bands, internal hernias, and/or bowel obstruction. This may in part be related to fear of patients to seek emergency care midst the pandemic as patients worry about contracting the infection from hospitals [26].

There has been some controversy over performing laparoscopic surgery during the pandemic due to the presumed risk of aerosolisation of viral particles, which has never been proven [27]. Some hospitals have set rules that only allow open surgery to be performed. For bariatric patients presenting with an emergency this can be challenging as the risks associated with open surgery including the need for ITU admission and pulmonary or wound-related problems, far outweigh the potential risk of viral aerosolisation. Fortunately, this survey found that in the rare circumstances where emergency surgery had to be performed, for the most part this was performed laparoscopically. Published guidelines exist on precautions needed intraoperatively to prevent potential aerosolization of the virus in the operation theatre [28].

When it comes to patient selection, the survey revealed that $60 \%$ of surgeons would give the highest priority to patients presenting with postoperative complications after bariatric surgery. This is not surprising as these patients would already be on semi-urgent lists before the pandemic. The next priority was given to type 2 diabetic patients which is expected as studies reported that the probability of hyperglycaemia remission depends on when the operation is performed during the diabetes' natural history [29]. The SOS study reported decreased rate of remission of type 2 diabetes in patients with 4 year history of the disease in comparison with patients with only 2 years history [30]. The least priority was given to patients with BMI $>60 \mathrm{~kg} /$ $\mathrm{m}^{2}$ perhaps because it was compared against other important priorities for bariatric surgeons who took the survey. The other indications that took priority over BMI $>60 \mathrm{~kg} / \mathrm{m}^{2}$, namely diabetes mellitus and multiple comorbidities, were associated with a higher risk for developing severe COVID19 complications. In addition, patients who need surgery to resolve complications of previous bariatric surgery had the top priority as these patients needed quick corrective intervention before developing more serious consequences.

How should patients be prioritised in the COVID-19 era is now the key question. The classic principle of prioritisation in the NHS is based on first-come-first-served rule, so patients who needs surgery are placed on a waiting list for elective surgery by turn.

Our survey reported results in contrary to this principle, as the responders neither prioritised patients according to their BMI nor to their turn in the waiting list, but instead according the actual clinical need. We believe that the usual principle of prioritisation should be revised at least during this unprecedented time.

Our results are in line with Rubino et al. [21] in their recent recommendations on prioritisation of access to bariatric surgery. They suggested that access to surgical treatment of bariatric and metabolic surgery should be based on disease-focussed clinical needs instead of BMI alone.

The authors specifically prioritise three groups of patients: firstly, those with $\mathrm{HbA} 1 \mathrm{c} \geq 8 \%$ on two or more oral medications, insulin use, history of cardiovascular disease, non-alcoholic steatohepatitis or two or more other metabolic conditions increasing cardiovascular risk, albuminuria or chronic kidney disease (stage 3 or 4 ) or $>5$ years of diabetes history. Secondly, patients with BMI $\geq 60 \mathrm{~kg} /$ $\mathrm{m}^{2}$ with more than two other metabolic conditions (other than type 2 diabetes) increasing cardiovascular risk, severe obesity hypoventilation syndrome, severe obstructive sleep apnoea, heart failure stage $\mathrm{C}$ or Chronic kidney (stage 3 or $4)$. The last group is patients who need weight loss surgery as a bridge to other operations which may be either lifesaving as organ transplant or to improve quality of life as hip replacement.

Recent guidelines published by the Federation of Surgical Specialty Association (FSSA) recommended similar approach. Urgent cases to be done either less than $24 \mathrm{~h}$ or $72 \mathrm{~h}$ depending on the clinical emergency. Elective cases to be done in less than 1 month if as part of cancer/transplant treatment, less than 3 months if significant/multiple end organ failure, to facilitate Musculoskeletal (MSK) physiotherapy or other surgeries as hernia, overdue balloon removal or revision to stop excessive weight loss [31].

Although starting with such high-risk patients appears to be more appealing to bariatric surgeons as surgery will be easier to justify to the NHS and the Clinical Commissioning Groups (CCGs), an alternative view is to start with low risk patients based on the fact that they are less liable 
to surgical complications and also perhaps less at risk of severe COVID-19 should they contract this in the peri- and postoperative period.

Another casualty of the COVID-19 pandemic is the compromised training opportunities for trainee surgeons. Very few consultants used e-training/simulations and for most trainees, there was no plan to compensate for the loss of training. This will likely have a significant impact on competence level of bariatric surgery trainees in the near future. The current pandemic is expected to negatively impact the provision of surgical education and training with unprecedented disruption in training programs and bariatric surgery programs are not an exception [32].

The survey was also sent to non-bariatric surgeons to ascertain their views about bariatric surgery recommencement after COVID-19. Unsurprisingly, there was a clear discrepancy between non-bariatric surgeons who classified it as low priority with no need to change commissioning criteria whilst most bariatric surgeons valued bariatric surgery as a medium to high priority with a need to change commissioning criteria to make access easier. We believe that the difference in sub-specialisation in surgery may create this type of discrepancy. Many non-bariatric surgeons consider bariatric surgery as non-urgent elective procedures that do not take top priority when compared to other domains including hernia surgery, breast surgery, trauma and emergency surgery and surgical oncology. According to this perspective, a patient with cancer should take the priority to prevent spread and a patient with hernia also should be prioritised since they can develop incarceration or strangulation of their hernia anytime. However, this perspective may not be accurate since patients indicated for bariatric surgery are also at high risk of developing serious morbidities in addition to being among the high-risk group for severe/critical COVID-19.

Limitations of this work include the low response rates which can be attributed to the short duration of the survey (1 week) during a period busy with emergency surgery, redeployment and many online meetings and webinars. Using surveys in research have their own limitations previously described such as skipped questions, personal agenda, accessibility issues and survey fatigue [33]. We believe that this is compensated by the diverse response from the highest volume bariatric surgery units around the UK.

\section{Conclusion}

This survey is an attempt to understand the impact of COVID-19 on UK bariatric surgery and the readiness of the specialty to restart. Also, it expresses the views of the bariatric surgeons regarding the restart plans.

\section{Compliance with ethical standards}

Disclosures Ahmed Ghanem, Sameh Emile, Jonathan Cousins, David Kerrigan, and Ahmed Rashid Ahmed have no conflicts of interest or financial ties to disclose.

\section{References}

1. Fontaine KR, Redden DT, Wang C, Westfall AO, Allison DB (2003) Years of life lost due to obesity. JAMA 289(2):187-193

2. Segula D (2014) Complications of obesity in adults: a short review of the literature. Malawi Med J 26(1):20-24

3. Gloy VL et al (2013) Bariatric surgery versus non-surgical treatment for obesity: a systematic review and meta-analysis of randomised controlled trials. BMJ 347:f5934

4. WHO, "WHO Timeline - COVID-19," 2020. https://www.who.int/ news-room/detail/27-04-2020-who-timeline---covid-19. Accessed 07 May 2020

5. Eng R (2020) COVID-19: good practice for surgeons and surgical teams-Royal College of Surgeons. https://www.rcseng.ac. uk/standards-and-research/standards-and-guidance/good-pract ice-guides/coronavirus/covid-19-good-practice-for-surgeons-andsurgical-teams/. Accessed 07 May 2020

6. Updated Intercollegiate General Surgery Guidance on COVID19-Royal College of Surgeons 2020. https://www.rcseng.ac.uk/ coronavirus/joint-guidance-for-surgeons-v2/. Accessed 19 Jun 2020

7. Peaple U (2016) Guidance for Clinical Commissioning Groups (CCGs): service specification guidance for obesity surgery. https ://www.england.nhs.uk/wp-content/uploads/2016/05/appndx-9serv-spec-ccg-guid.pdf. Accessed 14 Nov 2020

8. WHO (2020) Coronavirus disease (COVID-19) Situation Report-148. https://www.who.int/docs/default-source/coron aviruse/situation-reports/20200616-covid-19-sitrep-148-draft .pdf?sfvrsn=9b2015e9_2. Accessed 17 Jun 2020

9. NHS (2020) Next steps on NHS response to COVID-19. https ://www.england.nhs.uk/coronavirus/wp-content/uploads/sites /52/2020/03/urgent-next-steps-on-nhs-response-to-covid-19-lette r-simon-stevens.pdf. Accessed 20 May 2020

10. Jia H, Lubetkin EI (2005) The impact of obesity on health-related quality-of-life in the general adult US population. J Public Health (Bangkok) 27(2):156-164. https://doi.org/10.1093/pubmed/fdi02 5

11. Falagas ME, Kompoti M (2006) Obesity and infection. Lancet Infect Dis 6(7):438-446

12 Cai Q et al (2020) Obesity and COVID-19 severity in a designated hospital in Shenzhen, China. Diabetes Care 43:139

13. Simonnet A et al (2020) High prevalence of obesity in severe acute respiratory syndrome coronavirus-2 (SARS-CoV-2) requiring invasive mechanical ventilation. Obesity

14. Lighter $\mathrm{J}$ et al (2020) Obesity in patients younger than 60 years is a risk factor for Covid-19 hospital admission. Clin Infect Dis 9(10): 1093

15 Tamara A, Tahapary DL (2020) Obesity as a predictor for a poor prognosis of COVID-19: a systematic review. Diabetes MetabSyndrClin Res Rev 14:655

16. Docherty AB et al (2020) Features of 20133 UK patients in hospital with covid-19 using the ISARIC WHO Clinical Characterisation Protocol: prospective observational cohort study. bmj 369

17. Coronavirus: Emergency cash to help businesses, while operations delayed - BBC News (2020) https://www.bbc.co.uk/news/ uk-51933410. Accessed 19 Jun 2020 
18. Hollander JE, Carr BG (2020) Virtually perfect? Telemedicine for COVID-19. N Engl J Med 382(18):1679-1681

19. Official data demonstrates rapid rise of remote GP consultations I News I Health Service Journal (2020) https://www.hsj.co.uk/coron avirus/official-data-demonstrates-rapid-rise-of-remote-gp-consu ltations/7027540.article. Accessed 19 Jun 2020

20 Salari A, Shirkhoda M (2020) COVID-19 pandemic \& head and neck cancer patients management: The role of virtual multidisciplinary team meetings. Oral Oncol 105:104693

21. Rubino F et al (2020) Bariatric and metabolic surgery during and after the COVID-19 pandemic: DSS recommendations for management of surgical candidates and postoperative patients and prioritisation of access to surgery. Lancet Diabetes Endocrinol. 8:640

22. Garvey WT et al (2012) Two-year sustained weight loss and metabolic benefits with controlled-release phentermine/topiramate in obese and overweight adults (SEQUEL): a randomized, placebocontrolled, phase 3 extension study. Am J ClinNutr 95(2):297-308

23. Le Roux CW et al (2017) 3 years of liraglutide versus placebo for type 2 diabetes risk reduction and weight management in individuals with prediabetes: a randomised, double-blind trial. Lancet 389(10077):1399-1409

24. Collaborative C, Nepogodiev D, Bhangu A (2020) Elective surgery cancellations due to the COVID-19 pandemic: global predictive modelling to inform surgical recovery plans. Br J Surg 107:1440

25. Coronini-Cronberg S, John Maile E, Majeed A (2020) Health inequalities: the hidden cost of COVID-19 in NHS hospital trusts? J. R. Soc. Med. 113(5):179-184. https://doi.org/10.1177/01410 76820925230
26. WongLaura E, Hawkins Jessica E, MurrellKaren L (2020) Where are all the patients? Addressing Covid-19 fear to encourage sick patients to seek emergency care. NEJM Catal Innov Care Deliv

27. Emile SH (2020) Should we continue using laparoscopy amid the COVID-19 pandemic? Br J Surg

28. Zheng MH, Boni L, Fingerhut A (2020) Minimally invasive surgery and the novel coronavirus outbreak: lessons learned in China and Italy. Ann Surg

29. Still CD et al (2014) Preoperative prediction of type 2 diabetes remission after Roux-en-Y gastric bypass surgery: a retrospective cohort study. Lancet Diabetes Endocrinol 2(1):38-45

30. Sjöholm K et al (2015) Incidence and remission of type 2 diabetes in relation to degree of obesity at baseline and 2 year weight change: the Swedish Obese Subjects (SOS) study. Diabetologia 58(7):1448-1453

31. FSSA, Clinical Guide to Surgical Prioritisation During the Coronavirus Pandemic (2020) https://fssa.org.uk/_userfiles/pages/files/ covid19/prioritisation_master_240820.pdf. Accessed 10 Sep 2020

32. Al-Jabir A et al (2020) Impact of the coronavirus (COVID-19) pandemic on surgical practice-part 2 (surgical prioritisation). Int J Surg 79:233

33. Debois S (2019) 10 advantages and disadvantages of questionnaires: survey anyplace. https://surveyanyplace.com/questionna ire-pros-and-cons/. Accessed 23 Jun 2020

Publisher's Note Springer Nature remains neutral with regard to jurisdictional claims in published maps and institutional affiliations. 